\title{
CONCENTRACIÓN DE TITANIO EN TEJIDO RESPIRATORIO OBTENIDO DE AUTOPSIAS EN RESIDENTES DE LA CIUDAD DE MÉXICO
}

\author{
Adelaida Rayén Inés Manquián-Tejos ${ }^{1, a}$, Luis R. Tovar-Gálvez,b, Gabriel Yáñez-Canal2,o
}

\begin{abstract}
RESUMEN
Objetivos. Cuantificar la cantidad de titanio (Ti) en tejido respiratorio obtenidas por autopsia en personas que residieron en la Ciudad de México. Materiales y métodos. Se realizó un estudio exploratorio en el cual se obtuvieron 216 muestras de tejido respiratorio de lóbulos pulmonares y nódulos linfáticos peribronquiales, procedentes de autopsias médico legales de 36 cadáveres con antecedente de haber residido un mínimo de dos años en la Ciudad de México. Se realizaron análisis histopatológicos de las muestras y se cuantificó el Ti existente en ellas mediante plasma de inducción acoplado a la espectrofotometría de absorción atómica. Resultados. La cantidad de Ti en tejido respiratorio se distribuyó entre 0 microgramos de Ti en tejido seco ( $\mu \mathrm{g} \mathrm{Ti/g} \mathrm{ts)} \mathrm{y} \mathrm{39,7} \mu \mathrm{g} \mathrm{Ti/g}$ ts, se identificó la presencia de $\mathrm{Ti}$ distribuido hasta en seis veces mayor cantidad en los nódulos linfáticos que en lóbulos pulmonares. El análisis de conglomerados mostró que la muestra estaba conformada por cuatro grupos de individuos agrupados de acuerdo a la cantidad y distribución del Ti en el tracto respiratorio bajo, a la edad, índice de masa corporal, lugar y tiempo de residencia en la ciudad de México. Conclusiones. Debido al patrón de distribución observado el Ti encontrado en las muestras de tejido respiratorio podría provenir del aire urbano, la cantidad de Ti en conjunto con otros elementos podría ser causante de patologías respiratorias.
\end{abstract}

Palabras Clave: Enfermedades Respiratorias; Titanio; Toxicidad; Contaminación del aire; Material particulado. (Fuente: DeCS BIREME).

\section{CONCENTRATION OF TITANIUM IN RESPIRATORY TISSUE OBTAINED FROM AUTOPSIES IN MEXICO CITY RESIDENTS}

\begin{abstract}
Objetivos. To quantify the levels of titanium (Ti) in respiratory tissue obtained by autopsy in people who resided in Mexico City. Materials and Methods. An exploratory study was conducted in which 216 samples of respiratory tissue from pulmonary lobes and peribronchial lymph nodes were obtained from legal medical autopsies of 36 corpses with a minimum of two years of residence in Mexico City. Histopathological analyses of the samples were performed and the $\mathrm{Ti}$ existing in them was quantified by induction plasma coupled to atomic absorption spectrophotometry. Results. The amount of Ti in respiratory tissue was distributed between 0 micrograms of Ti in dry tissue ( $\mu \mathrm{g} \mathrm{Ti} / \mathrm{g}$ ts) and $39.7 \mu \mathrm{g} \mathrm{Ti} / \mathrm{g}$ ts; the presence of $\mathrm{Ti}$ was identified as distributed in up to six times a higher quantity in the lymph nodes than in pulmonary lobes. Cluster analysis showed that the sample consisted of four groups of individuals grouped according to the amount and distribution of $\mathrm{Ti}$ in the lower respiratory tract, age, body mass index, place and time of residence in Mexico City. Conclusions. Due to the distribution pattern observed, Ti found in respiratory tissue samples may come from urban air; the amount of $\mathrm{Ti}$ in conjunction with other elements may be the cause of respiratory pathologies.
\end{abstract}

Keywords: Respiratory Tract Diseases; Titanium, toxicity, air pollution, particulate matter (source: MeSH NLM).

\footnotetext{
Centro Interdisciplinario de Investigaciones y Estudios sobre Medio Ambiente y Desarrollo (CIIEMAD). Instituto Politécnico Nacional. México.

Universidad Industrial de Santander. Colombia.

Obstetra, Ph.D; ${ }^{\mathrm{b}}$ Ingeniero Químico. Ph.D.; ${ }^{\mathrm{c}}$ Matemático. Ph.D

Los resultados del presente estudio son parte de la tesis de doctorado de Adelaida Manquián, titulada «Contenido de Metales en el Tracto Respiratorio Bajo de Personas que Residieron en la Ciudad de México» Año 2010

Recibido: 02/09/2018 Aprobado: 13/03/2019 En línea: 28/06/2019
}

Citar como: Manquián-Tejos ARI, Tovar-Gálvez LR, Yáñez-Canal G. Concentración de titanio en tejido respiratorio de personas expuestas a la contaminación del aire. Rev Peru Med Exp Salud Publica. 2019;36(2):247-54. doi: http://dx.doi.org/10.17843/rpmesp.2019.362.3900. 


\section{INTRODUCCIÓN}

El conocimiento actual sobre la toxicidad del dióxido de titanio (Ti) proviene de estudios experimentales en vivo e in vitro ${ }^{(1-5)}$, en los que se informa que el óxido de titanio $\left(\mathrm{TiO}_{2}\right)$ puede ser absorbido principalmente a través del tracto digestivo, respiratorio y, dependiendo del tamaño de las partículas, llegar al torrente circulatorio y ser distribuido y acumulado en hígado, bazo, riñón, pulmón, ganglios $^{(6)}$ y cerebro ${ }^{(2,7,8)}$.

La inhalación de Ti puede causar inflamación, citotoxicidad y genotoxicidad ${ }^{(2,3)}$, por otro lado, existe consenso entre los investigadores respecto al efecto dosis respuesta entre dosis inhalada de $\mathrm{TiO}_{2}$ y proceso inflamatorio ${ }^{(2)}$. Los efectos citotóxicos se asocian con la forma de las partículas ${ }^{(5)}$ y con el área de superficie de las partículas, siendo las nanopartículas más citotóxicas que las partículas finas ${ }^{(9)}$. El efecto genotóxico del $\mathrm{TiO}_{2}$ sigue siendo controvertido ${ }^{(2,10)}$ a pesar de resultados de investigación que muestran efectos mutagénicos, alteraciones mitóticas, daño genético y daño al ADN mediado por oxidantes que dan lugar a apoptosis celular e inducción de micronúcleos, en células de animales experimentales expuestos $\mathrm{a} \mathrm{TiO}_{2}$. ${ }^{(11)}$

Entre los hallazgos patológicos pulmonares asociados con la inhalación de $\mathrm{TiO}_{2}$ se han descrito la exacerbación de enfermedades alérgicas como el asma, enfermedad pulmonar obstructiva crónica, rinosinusitis crónica ${ }^{(12)}$, neumonías ${ }^{(4)}$, gran reacción inflamatoria persistente ${ }^{(13)}$, daño pulmonar, fibrosis, enfisema ${ }^{(14)}$, defectos en la función de los macrófagos ${ }^{(15,16)}$ y cáncer de pulmón ${ }^{(2)}$.

En trabajadores expuestos ocupacionalmente al $\mathrm{TiO}_{2}$ y $\mathrm{TiO}_{4}$ se han observado cambios pulmonares caracterizados por disminución de la capacidad ventilatoria, enfermedad pleural ${ }^{(17)}$, fibrosis, bronconeumonía, numerosos macrófagos con abundantes depósitos de pigmentos blancos conteniendo una gran cantidad de $\mathrm{Ti}{ }^{(18)}$ y lesiones granulomatosas ${ }^{(19)}$. La gravedad de las patologías se asocia con el tiempo de exposición en manufacturas de $\mathrm{Ti}^{(17)}$. De otro lado, un estudio de cohorte que incluyó 3607 trabajadores expuestos al $\mathrm{TiO}_{2}$ (seguidos desde 1935 al 2006), no mostró asociación positiva entre exposición ocupacional y enfermedades respiratorias, así como tampoco con enfermedades cardiacas, mortalidad y cáncer de pulmón ${ }^{(20)}$.

El riesgo cancerígeno del $\mathrm{TiO}_{2}$ en humanos fue reevaluado en febrero del 2006 por un Grupo de Trabajo de la IARC (International Agency for Research on Cancer), concluyendo que a pesar de que los resultados de los estudios epidemiológicos realizados en industrias no aportan suficientes pruebas de carcinogenicidad, existe suficiente evidencia de su efecto carcinogénico en roedores, por lo que el $\mathrm{TiO}_{2}$ fue clasificado como posiblemente carcinógeno para los humanos ${ }^{(21,22)}$.

\section{MENSAJES CLAVE}

Motivación para realizar el estudio. En Ciudad de México se ha reportado presencia de titanio en las partículas del aire urbano, lo que causa preocupación debido a que se ha demostrado que este elemento daña la salud respiratoria de la población expuesta.

Principales hallazgos. Observamos presencia de titanio en muestras de tejido respiratorio, cuya cantidad muestra correlación con enfisema pulmonar, con la edad, así como del tiempo de residencia.

Implicancias. Un estricto control ambiental de las emisiones de titanio puede beneficiar la salud respiratoria de la población expuesta en las grandes ciudades.

Estos antecedentes causan preocupación debido a que en las últimas décadas se ha incrementado el uso del $\mathrm{TiO}_{2}$ en la industria y la nanotecnología ${ }^{(23)}$ y con ello la exposición humana en ambientes urbanos, ${ }^{(2)}$ en especial en megaciudades con problemas de contaminación del aire como Ciudad de México, donde se sabe que los metales, incluido el $\mathrm{Ti}$, son componentes importantes de las partículas ${ }^{(24,25)}$. Se ha reportado que la presencia y la cantidad de los diferentes metales encontrados en las partículas varían de acuerdo con la zona geográfica ${ }^{(24)}$, y con la época del año; las $\mathrm{PM}_{10}$ transportan mayor cantidad de $\mathrm{Ti}$ que las $\mathrm{PM}_{2,5}{ }^{(26)}$. En líneas celulares ${ }^{(27)}$, así como en la población expuesta, en especial los niños, se han observado procesos inflamatorios en grados que varían según el lugar de residencia y el tiempo de exposición ${ }^{(7)}$, desconociéndose si el $\mathrm{Ti}$, elemento no esencial para la vida humana, se acumula en el tejido respiratorio de la población expuesta a la contaminación del aire en la ciudad de México. El objetivo del presente estudio fue identificar la presencia de $\mathrm{TiO}_{2}$ en muestras de tejido respiratorio de personas que residieron en la Ciudad de México.

\section{MATERIALES Y MÉTODOS}

\section{DISEÑO Y POBLACIÓN DE ESTUDIO}

Se realizó una investigación de tipo exploratorio, que integró un estudio histopatológico y otro químico en el cual se evaluaron la presencia de 23 elementos metálicos. El análisis aislado del Ti se realizó el 2017. La población de estudio estuvo conformada por individuos fallecidos por muerte accidental a quienes se les realizó autopsia médico legal en el Servicio Médico Forense de la Ciudad de México (SEMEFO) y se obtuvo muestras de tejido respiratorio provenientes de cada lóbulo pulmonar y del nódulo linfático peribronquial. Se incluyeron individuos con antecedente de residencia en la Ciudad de México por dos o más años y se excluyeron cadáveres con historia haber sido fumadores o expuestos laboralmente a la inhalación de metales o que hayan estado embarazadas. 
Los antecedentes se obtuvieron desde la ficha clínica médico legal y a través de una entrevista personal a un familiar del fallecido y se agruparon en sociales, biológicos y de exposición ambiental. Los sociales hacen referencia a género, edad, ocupación y escolaridad; los biológicos a enfermedades cardiorrespiratorias y otras patologías, uso de drogas, causa de muerte y el índice de masa corporal (IMC); los antecedentes de exposición ambiental considerados fueron lugar de nacimiento, lugar de residencia, tiempo de residencia en la ciudad de México, tráfico vehicular circundante a su vivienda, ubicación del lugar de trabajo, tiempo de permanencia en el lugar de trabajo, tiempo en ambientes extramuros, tiempo en ambientes intramuros, tiempo empleado en movilizarse y percepción de riesgo de exposición.

\section{RECOLECCIÓN Y ANÁLISIS DE MUESTRAS}

Durante la recolección de las muestras, y para evitar la contaminación, se utilizó un bisturí de vidrio y cada muestra (15 g de tejido húmedo) se dividió en dos submuestras, una se preparó para el estudio químico y la otra para el estudio histopatológico.

Las muestras se mantuvieron a $4{ }^{\circ} \mathrm{C}$ antes de ser procesadas. Posteriormente, las diversas secciones anatómicas del tejido pulmonar (lóbulos pulmonares y nódulos peribronquiales) se pesaron y secaron a $85 \pm 5^{\circ} \mathrm{C}$ en horno de convección forzada durante 24 horas. El tejido seco se pesó y cada muestra se colocó en un vial de vidrio debidamente etiquetado donde fue triturado utilizando un émbolo del mismo material. Durante todo el procedimiento se tomaron precauciones para evitar la contaminación de las muestras, principalmente por metales.

Para eliminar la absorbancia debida a detergentes, a muestras y a patrones de referencia, todos los recipientes de vidrio y polietileno utilizados se lavaron y se mantuvieron sumergidos durante 48 horas en una solución acuosa de ácido nítrico al $2 \%$; posteriormente fueron enjuagados varias veces con agua ultrapura. El agua que se utilizó durante la preparación de todas las soluciones fue agua doblemente destilada y purificada adicionalmente con un sistema Milli-Q (Millipore, Bedford, MA, EE. UU.) Se usó ácido nítrico (65\%) y ácido clorhídrico (37\%), ambos J.T.Baker suprapuros, para la digestión de las muestras.

Para la digestión de las muestras se utilizó un horno microondas Perkin Elmer modelo Para Physica (Graz, Austria) que proporcionó microondas a una frecuencia de $2,45 \mathrm{GHz}$, equipado con recipientes de digestión de teflón. En cada recipiente de teflón se colocó $0,6000 \mathrm{~g}$ de tejido seco, añadiéndose $4,0 \mathrm{ml}$ de $\mathrm{HNO} 3$ y $0,5 \mathrm{ml}$ de $\mathrm{HCL}$, ambos concentrados y ultrapuros. En cada secuencia de digestión se preparó una muestra «blanco» conteniendo sólo los ácidos minerales. La selección del programa USER002M se realizó posterior a su validación utilizando diversas matrices (tejido animal) y programas de digestión.
La temperatura más alta alcanzada fue de $210^{\circ} \mathrm{C}$, la presión dentro del recipiente de digestión fue de aproximadamente 30 bares y la potencia fue de 1000 vatios. Este método proporcionó una calidad de descomposición óptima, la que se logró cuando se obtuvo una solución cristalina con efectos mínimos sobre la matriz. Luego, cada muestra digerida, incluidas las muestras «blanco», fueron filtradas utilizando papel filtro Watman 41 y aforadas en un matraz volumétrico a $25 \mathrm{ml}$ con agua deionizada (Milli-Q, Millipore, Bedford, MA). Cada muestra digerida se almacenó en una botella de plástico hasta el análisis. Las muestras se mantuvieron a $4{ }^{\circ} \mathrm{C}$.

\section{CUANTIFICACIÓN DE TITANIO}

Las muestras fueron analizadas de acuerdo con las especificaciones del fabricante en un espectrómetro de plasma de inducción acoplado a la espectrofotometría de absorción atómica (ICP-OES) modelo Optima 4300DV (PerkinElmer). Las condiciones operativas del espectrómetro para el Ti fueron: longitud de onda: 334,94 II nm y el rango de la concentración del estándar fue de 0.1 - 10 ppm. La optimización y curva de calibración se controlaron desde el computador a través de programas ya establecidos, se midieron los límites de detección del instrumento $(0,02 \mu \mathrm{g} / \mathrm{L})$ del método $(0,04 \mu \mathrm{g} / \mathrm{L})$ y el porcentaje de recuperación $(79,68 \%)$, con el objeto de validar los resultados. Se utilizó un estándar (Perkin Elmer) para construir la curva de calibración [(1) blanco; (S1) 0,1 mg / L de Ti; (S2) $1 \mathrm{mg} / \mathrm{L}$ de Ti; (S3) $10 \mathrm{mg} / \mathrm{L}$ Ti]. En todos los casos, se realizaron tres lecturas para cada muestra o estándar.

\section{ANÁLISIS HISTOPATOLÓGICO}

Fue realizado en el SEMEFO, cuyo laboratorio de patología está acreditado por la entidad mexicana de acreditación (EMA). Las muestras fueron secadas y dejadas en formol al $10 \%$ por tres días, posterior a lo cual se colocaron en celdas de inclusión, en cortes de 2 x $0.5 \mathrm{~cm}$. La deshidratación de las muestras se hizo en un procesador Jung Histokinette 2000 marca Leica automático. Para la inclusión de las muestras en parafina se utilizó un equipo Histoembedder Tissutec marca Leica. Del bloque de parafina conteniendo la muestra se hicieron cortes de cuatro a cinco $\mu \mathrm{m}$ con un equipo Jung Histocut marca Leica. Posteriormente, se prepararon baños de flotación y rehidratación de las muestras y una vez que estuvo fija en la laminilla de vidrio se sometió a tinción (eosina-hematoxilila) y se cubrió con cubre objeto previo a ser etiquetada. Se realizaron ocho lecturas de cada muestra utilizando un microscopio Zeiss binocular de cinco objetivos modelo Axioplan. Los diagnósticos obtenidos fueron globales.

\section{ANÁLISIS ESTADÍSTICO}

El análisis estadístico contempló cinco etapas: 1) análisis exploratorio de los datos que permitió identificar los valores 
atípicos los cuales fueron excluidos del estudio; 2) test $t$ para corroborar que las cantidades de Ti encontrados en los lóbulos y nódulos no son resultado del azar y test de normalidad de los datos; 3 ) análisis de varianza intrasujetos con la prueba no paramétrica de Friedman para contrastar la igualdad de las medianas en la concentración de Ti en los diferentes segmentos respiratorios; 4) test no paramétrico $U$ de Mann-Whitney para contrastar la cantidad de Ti en lóbulos y nódulos en personas con patologías respiratorias; 5) se utilizó un método de análisis de conglomerados (cluster analysis) con el objeto de establecer grupos homogéneos de individuos de acuerdo a las variables consideradas. El análisis realizado en SPAD 4.5 se basó en los ocho factores obtenidos en un análisis previo de componentes principales (ACP) que explicaban el $83,0 \%$ de la variabilidad existente al considerar todas las variables. Se utilizó el criterio de varianza mínima de Ward con distancia euclidiana. Los softwares estadísticos utilizados fueron SPSS 18, SPAD 4.5 y Excel.

\section{CONSIDERACIONES ÉTICAS}

Se obtuvo la aprobación del protocolo de investigación por el Comité de Ética del SEMEFO y la autorización legal otorgada por el Honorable Consejo de la Judicatura del Distrito Federal de México.

\section{RESULTADOS}

Se tuvo acceso a la autopsia médico legal de 36 individuos (24 hombres y 12 mujeres), con una edad promedio de 44,7 años (rango 3 a 82 años) que habían residido en la ciudad de México un tiempo promedio de 23,6 años (rango 2 a 82 años). Se analizaron 216 muestras de tejido respiratorio provenientes de cada lóbulo pulmonar y del nódulo linfático peribronquial.

La cantidad de Ti muestra gran variabilidad, los valores se distribuyen entre 0 microgramos de Ti en tejido seco ( $\mu \mathrm{g} \mathrm{Ti/g} \mathrm{ts)} \mathrm{y} \mathrm{39,7} \mu \mathrm{g} \mathrm{Ti/g} \mathrm{ts,} \mathrm{observándose} \mathrm{cantidades}$ de Ti, cinco y hasta seis veces más altas en los nódulos peribronquiales (Md 6,8 $\mu \mathrm{g} \mathrm{Ti/g} \mathrm{ts)} \mathrm{comparados} \mathrm{con}$ los valores de $\mathrm{Ti}$ cuantificados en lóbulos pulmonares (Md entre 1,0 y 1,9 $\mu \mathrm{g} \mathrm{Ti/g}$ ts), los test de normalidad de Shapiro-Wilk y Anderson-Darling $(p<0,02)$ rechazan la normalidad de las distribuciones de las cantidades de $\mathrm{Ti}$ en la Figura 1 se muestran cada uno de los segmentos respiratorios analizados que dan cuenta de la asimetría de las distribuciones de la cantidad de Ti.

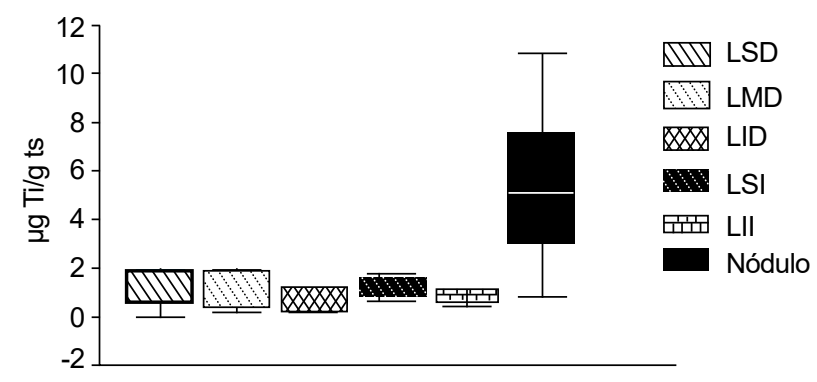

Figura 1. Microgramos de $\mathrm{Ti}$ en tejido seco ( $\mu \mathrm{g} \mathrm{Ti} / \mathrm{g}$ ts) encontrados en lóbulos pulmonares superiores derecho (LSD) e izquierdo (LII), lóbulo medio derecho (LMD), lóbulos inferiores derecho (LID), lóbulos inferiores izquierdo (LII) y los nódulos linfáticos peribronquiales.

La prueba no paramétrica de Friedman mostró que las diferencias son estadísticamente significativas al considerar los seis tejidos ( $p=0,001)$; al excluir los nódulos la prueba ya no fue significativa $(p=0,386)$ por lo que la concentración de Ti en los lóbulos es semejante, y permitió considerar un solo nivel promedio de concentración de Ti en los lóbulos.

Como se observa en la Tabla 1, a mayor edad la concentración de $\mathrm{Ti}$ en los pulmones se incrementa, asimismo la concentración de Ti en los nódulos es mayor en las personas con menor IMC; y entre más tiempo de residencia en Ciudad de México más alta la concentración de Ti en los nódulos.

Las patologías respiratorias encontradas fueron enfisema, hiperplasia, antracosis, edema pulmonar, edema alveolar y neumonía. Al comparar las personas con patologías respiratorias con las personas que no tenían, sólo el enfisema presentó diferencias en la mediana de concentración de Ti en los lóbulos $(p=0,020)$.

La edad se correlacionó con la cantidad de Ti en los lóbulos en las personas con edema alveolar (Rho 0,784, $p=0,001$ ), edema pulmonar (Rho 0,619, $p=0,014$ ), neumonía (Rho $0,633, p=0,006$ ) e hiperplasia nodular (Rho 0,508, $p=0,016$ ). EI IMC se correlacionó significativamente con la cantidad de $\mathrm{Ti}$ en los lóbulos en las personas con antracosis $(0,888$, $p=0,003$ ). El tiempo de residencia en la Ciudad de México

Tabla 1. Correlaciones entre la cantidad de titanio en lóbulos y nódulos con variables biológicas y de exposición ambiental

\begin{tabular}{|c|c|c|c|}
\hline & Edad & Índice de masa corporal & Tiempo de residencia en la Ciudad de México \\
\hline & Rho (Valor de p) & Rho (Valor de p) & Rho (Valor de p) \\
\hline Titanio en lóbulos & $0,458(0,006)$ & ND & ND \\
\hline Titanio en nódulos & $0,474(0,004)$ & $-0,339(0,046)$ & $0,397(0,018)$ \\
\hline
\end{tabular}

ND: no disponible 
correlacionó significativamente con la cantidad de Ti en los lóbulos y nódulos en las personas con edema pulmonar (Rho 0,542, p=0,037) y neumonía (Rho 0,526, p=0,030) respectivamente.

El análisis de conglomerados permitió agrupar variables con la máxima homogeneidad en cada grupo y mayor diferencia entre los grupos. Las variables que establecieron la clasificación fueron: la cantidad de Ti acumulado en los lóbulos pulmonares y nódulos peribronquiales, edad, tiempo y el lugar de residencia en la ciudad de México, tiempo de permanencia en ambiente intramuros y extramuros, horas de locomoción, estado nutricional y presencia de patologías.

El grupo 1 y 2 incluye los casos de menor edad de la muestra (medias 38 y 40 años respectivamente), con las concentraciones más bajas de Ti en lóbulos y nódulos y estado nutricional normal. En ambos grupos el tiempo de permanencia en ambientes extramuros es superior a la media muestral (7 y 9 horas respectivamente), el primer grupo incorpora, además, tiempo de uso de locomoción. Con relación al lugar de residencia y trabajo, todos los casos en el primer grupo habían residido y trabajado en la zona centro de la ciudad durante un tiempo inferior a la media muestral; en el segundo grupo el $89 \%$ de los casos, habían residido en el sur y $78 \%$ trabajado en el sur de la ciudad. Sólo el primer grupo incluyó casos con diagnóstico de patologías, $86 \%$ se diagnosticó con edema alveolar. EI tercer grupo incluyó igual proporción de hombres y mujeres, todos trabajaban y residían en la zona norte de la ciudad por 28 años, el $88 \%$ de ellos tenía un estado nutricional normal y el porcentaje restante bajo peso, la edad media era 49 años, un $67 \%$ de los casos tenía diagnóstico de edema pulmonar, neumonía e hiperplasia nodular y cantidad de Ti en lóbulos y nódulos peribronquiales superior a la media muestral. El cuarto grupo quedó conformado por hombres mayores de 58 años, residentes de la zona norte de la ciudad por 31 años y más, trabajaban en el centro y norte de la ciudad, $57 \%$ de ellos tenía mayor IMC y el porcentaje restante IMC bajo, pasaban mayor tiempo en ambientes intramuros, empleaban más de una hora en locomoción, presentaban las concentraciones más altas de Ti en lóbulos y nódulos peribronquiales, más del $70 \%$ de los casos tenía diagnóstico de neumonía (Tabla 2).

\section{DISCUSIÓN}

El estudio muestra que las personas que han residido en Ciudad de México por más de dos años pueden acumular $\mathrm{Ti}$ en los lóbulos pulmonares y ganglios linfáticos peribronquiales en cantidades variables; este comportamiento ha sido descrito para metales no esenciales para la vida humana, los que al no cumplir con ninguna función biológica se comportan como contaminantes y sus niveles son controlados externamente, de tal manera que su patrón de distribución puede ser similar al de los niveles ambientales ${ }^{(28)}$, a diferencia del comportamiento de los elementos esenciales que al estar sujetos a un control interno muestran una distribución normal ${ }^{(28,29)}$.

Llama la atención la alta concentración de Ti encontrada en los ganglios linfáticos peribronquiales en comparación con la concentración encontrada en los cinco lóbulos pulmonares; hallazgo que podría tener relación con la falla en el mecanismo de limpieza descrito por algunos investigadores que reportan que las partículas, en especial

Tabla 2. Valores promedios en los grupos de conglomerados de las variables cuantitativas

\begin{tabular}{lcccccccc}
\hline & $\mathbf{N}$ & $\begin{array}{c}\text { Lóbulos } \\
(\mu \mathrm{g} / \mathrm{g} \text { ts) }\end{array}$ & $\begin{array}{c}\text { Nódulos } \\
(\mu \mathrm{g} / \mathrm{g} \text { ts) }\end{array}$ & $\begin{array}{c}\text { Locomoción } \\
\text { (horas) }\end{array}$ & $\begin{array}{c}\text { Tiempo } \\
\text { Extramuros } \\
\text { (horas) }\end{array}$ & $\begin{array}{c}\text { Tiempo } \\
\text { Intramuros } \\
\text { (horas) }\end{array}$ & $\begin{array}{c}\text { Edad } \\
\text { (años) }\end{array}$ & $\begin{array}{c}\text { Tiempo } \\
\text { Residencia }\end{array}$ \\
\hline Grupo 1 & 14 & 1,62 & 12,28 & 1,6 & 7,2 & 16,7 & 38,4 & 20,3 \\
\hline Grupo 2 & 9 & 3,13 & 9,73 & 0,9 & 9,3 & 15,5 & 40,7 & 30,8 \\
Grupo 3 & 6 & 2,30 & 12,79 & 0.7 & 4,5 & 19,5 & 49,2 & 28,5 \\
\hline Grupo 4 & 7 & 2,66 & 16,73 & 1,7 & 4,8 & 19,1 & 58,6 & 31,4 \\
$\begin{array}{l}\text { Promedios } \\
\text { totales }\end{array}$ & 36 & 2,31 & 12,59 & 1,3 & 6,8 & 17,4 & 44,7 & 26,4 \\
\hline
\end{tabular}

Grupo 1: menores de edad de la muestra, concentraciones más bajas de Ti en lóbulos y nódulos y estado nutricional normal, tiempo de permanencia en ambientes extramuros superior a la media muestral, tiempo de uso de locomoción residido y trabajado en la zona centro de la ciudad durante un tiempo inferior a la media muestral, con diagnóstico de patologías respiratorias siendo $86 \%$ con diagnosticó con edema alveolar.

Grupo 2: menores de edad de la muestra, concentraciones más bajas de Ti en lóbulos y nódulos y estado nutricional normal tiempo de permanencia en ambientes extramuros es superior a la media muestral, $89 \%$ de los casos, habían residido en el sur y $78 \%$ trabajado en el sur de la ciudad,

Grupo 3: incluyó igual proporción de hombres y mujeres, todos trabajaban y residían en la zona norte de la ciudad por 28 años, $88 \%$ tenía un índice de masa corporal normal y el porcentaje restante menor, la edad media era 49 años, un $67 \%$ de los casos tenía diagnóstico de edema pulmonar, neumonía e hiperplasia nodular y cantidad de Ti en lóbulos y nódulos peribronquiales superior a la media muestral.

Grupo 4: conformado por hombres mayores de 58 años, residentes de la zona norte de la ciudad por 31 años y más, trabajaban en el centro y norte de la ciudad, $57 \%$ de ellos tenía mayor IMC y el porcentaje restante IMC bajo, pasaban mayor tiempo en ambientes intramuros, empleaban más de una hora en locomoción, presentaban las concentraciones más altas de Ti en lóbulos y nódulos peribronquiales, más del $70 \%$ de los casos tenía diagnóstico de neumonía. 
las ultrafinas ( $\leq 20 \mathrm{~nm}$ de $\mathrm{TiO}_{2}$ ), inducen daño de la función de los macrófagos ${ }^{(1,15)}$ y dependiendo de la magnitud del daño, las partículas pueden llegar rápidamente a las células epiteliales y ser transportadas al espacio subepitelial ${ }^{(30,31)}$ y a los ganglios linfáticos, ${ }^{(32)}$ siendo retenidas así en el tejido respiratorio ${ }^{(33)}$. Este mismo argumento, sumado al hecho de que los individuos del estudio estuvieron expuestos a respirar aire contaminado por partículas cuya composición química varía dependiendo de las diferentes sub áreas de la Ciudad de México ${ }^{(24,25)}$, podría explicar la gran variabilidad en la distribución de las cantidades de Ti cuantificadas entre los individuos del estudio.

Los resultados de investigaciones que muestran efectos de la contaminación del aire y efectos de elementos contenidos en las partículas ${ }^{(7,27)}$ destacan la asociación que tienen las diferentes sub áreas de la Ciudad de México con la gravedad de los efectos, por ejemplo, se ha observado que las partículas PM 10 provenientes de la zona norte son más citotóxicas que las partículas del sur de la ciudad ${ }^{(27)}$, lo que muestra la importancia de incorporar la variable lugar de residencia en la Ciudad de México en estudios que busquen riesgos o efectos perjudiciales de la población expuesta a la contaminación del aire.

En este estudio, el lugar de residencia fue una de las variables que permitió establecer la clasificación de grupos en el análisis de conglomerados, llamando la atención el grupo de individuos con las cantidades más altas de $\mathrm{Ti}$ en nódulos peribronquiales así como en lóbulos, habían sido residentes de la zona norte de la ciudad, que es precisamente la zona industrial, donde las partículas contienen mayor cantidad de metales, incluido el $\mathrm{Ti}^{(24,26)}$, indicando que a mayor dosis de exposición mayor es la cantidad de elemento retenido en el tejido respiratorio ${ }^{(32,33)}$.

En poblaciones expuestas a ambientes urbanos contaminados, se sabe que, a pesar de observarse algunas asociaciones entre enfermedades respiratorias y presencia de elementos metálicos, es difícil afirmar que tales efectos se deban exclusivamente al Ti retenido, ya que se encuentra formando parte de una masa heterogénea compuesta de muchos elementos, incluidos los metales ${ }^{(2)}$. A pesar de esta observación, destacamos que en el presente estudio se ha observado una correlación significativa entre la presencia de Ti en lóbulos con enfisema pulmonar, además uno de los casos con enfisema, mostró la cantidad más elevada de $\mathrm{Ti}$ en nódulos peribronquiales de toda la muestra, esto llama la atención si se tiene en cuenta los resultados de un estudio experimental que reporta que el Ti produce enfisema ${ }^{(14)}$.

No encontramos reportes que muestren la intervención de variables biológicas y sociales que se asocien con el riesgo de enfermar por efecto de la retención de $\mathrm{Ti}$ en el tejido respiratorio. A pesar de contar sólo con una aproximación del estado nutricional, observamos que el grupo con mayor cantidad de Ti en lóbulos y nódulos peribronquiales, tenía en proporciones similares, índice de masa corporal menor y mayor.

Finalmente, por la misma forma en que se llevó a cabo el estudio, presenta algunas limitaciones que dificultan inferir los resultados para las poblaciones de las cuales provenían los individuos estudiados. Además del pequeño tamaño muestral y de la imposibilidad de realizar una selección aleatoria, se tuvo que recurrir a fuentes secundarias para recolectar la información de las variables biológicas y sociales. Adicionalmente, se presenta la imposibilidad de comparar los resultados del estudio químico con otros similares, debido a la falta de estudios y de consenso de los laboratorios sobre los métodos de cuantificación y los estándares a utilizar ${ }^{(34,35)}$. A pesar de estas limitantes los resultados pueden ser considerados por la autoridad sanitaria y ambiental como una alerta para vigilar y controlar las emisiones de la industria y del uso de la nanotecnología.

En conclusión, el Ti encontrado en las muestras de tejido respiratorio puede provenir del aire urbano debido al patrón de distribución observado, retenido principalmente en los nódulos peribronquiales, la cantidad de Ti encontrado podría ser causante, en conjunto con otros elementos, de patologías tales como enfisema pulmonar con la cual se observó diferencias significativas. Se puede deducir además que las personas que residen por más de dos años en Ciudad de México, retienen Ti en cantidades muy variables, distribuyéndose de manera heterogénea, dependiendo de características biológicas como la edad y el estado nutricional así como de variables de exposición ambiental, principalmente del tiempo y lugar de residencia en la ciudad.

Agradecimientos: Al Servicio Médico Forense del D.F. (SEMEFO) por las facilidades otorgadas en la etapa de muestreo. A la Dra. Elena Piña jefa del laboratorio de patología del SEMEFO por el aporte en el análisis de las muestras. A Lorena Campos guía del estudio químico de las muestras A Ciro Márquez por el préstamo de equipo (ICP) y al Doctor Álvaro Osornio por sus importantes aportes.

Contribución de los autores: ARIMT, LRTG y GYC participaron en la concepción, redacción y aprobación de la versión final del artículo. Durante la investigación ARIMT participó en todas las etapas. LRTG en el diseño de técnicas y procedimientos de laboratorio y GYC en la recolección de información y análisis estadístico de los datos.

Fuentes de financiamiento. La investigación fue financiada por Instituto Politécnico Nacional de México a través del Programa Institucional de investigadores (PIFI).

Conflictos de interés. Los autores declaran no tener conflictos de intereses en la publicación del artículo. 


\section{REFERENCIAS BIBLIOGRÁFICAS}

1. Oberdörster G, Finkelstein JN, Johnston C, Gelein R, Cox C, Baggs R, et al. Acute pulmonary effects of ultrafine particles in rats and mice. Res Rep Health Eff Inst. 2000; 95:5-74.

2. Shi H, Magaye R, Castranova V, Zhao J. Titanium dioxide nanoparticles: a review of current toxicological data. Part Fibre Toxicol. 2013; 10: 1-33.

3. Chen T, Yan J, Li Y. Genotoxicity of titanium dioxide nanoparticles. J Food Drug Anal. 2014; 22: 95-104.

4. Hashiguchi $S$, Yoshida $H$, Akashi T, Komemoto K, Ueda T, Ikarashi Y, et al. Titanium dioxide nanoparticles exacerbate pneumonia in respiratory syncytial virus (RSV)-infected mice. Environ Toxicol Pharmacol. 2015;39(2):879-86.

5. Hattori $\mathrm{K}$, Nakadate $\mathrm{K}$, Morii A, Noguchi T, Ogasawara Y, Ishii K. Exposure to nano-size titanium dioxide causes oxidative damages in human mesothelial cells: The crystal form rather than size of particle contributes to cytotoxicity. Biochem Biophys Res Commun. 2017; 492(2):218-223.

6. Gutiérrez E, Pérez JA, Márquez SG, Delgado NL, Chirino YI, Iglesias GG, et al. Dióxido de titanio nanopartículas inducen fuerte estrés oxidativo y el daño mitocondrial en las células gliales. Free Radic Biol Med. 2014;10: 84-94.

7. Calderón-Garcidueñas L, ReynosoRobles R, Pérez-Guillé B, Mukherjee PS, Gónzalez-Maciel A. Combustion-derived nanoparticles, the neuroenteric system, cervical vagus, hyperphosphorylated alpha synuclein and tau in young Mexico City residents. Environ Res. 2017;159:186201.

8. Allen JL, Oberdorster G, MorrisSchaffer K, Wong C, Klocke C, Sobolewski M, et al. Developmental neurotoxicity of inhaled ambient ultrafine particle air pollution: Parallels with neuropathological and behavioral features of autism and other neurodevelopmental disorders. Neurotoxicology. 2017; 59:140-154.

9. Oberdörster G. Pulmonary effects of inhaled ultrafine particles. Int Arch Occup Environ Health. 2001; 74: 1-8.

10. Warheit DB, Donner EM. Risk assessment strategies for nanoscale and fine-sized titanium dioxide particles:
Recognizing hazard and exposure issues. Food Chem Toxicol. 2015;85:138-47.

11. Rahman Q, Lohani M, Dopp E, Pemsel H, Jonas L, Weiss DG, Schiffmann D. Evidence that ultrafine titanium dioxide induces micronuclei and apoptosis in Syrian hamster embryo fibroblasts. Environ Health Perspect. 2002;110:797- 00 .

12. Jonasson S, Gustafsson A, Koch B, Bucht A. Inhalation exposure of nano-scaled titanium dioxide ( $\mathrm{TiO} 2)$ particles alters the inflammatory responses in asthmatic mice. Inhal Toxicol. 2013; 25:179-91.

13. Tomioka H, Kaneda T, Katsuyama E, Kitaichi M, Moriyama H, Suzuki E. Elemental analysis of occupational granulomatous lung disease by electron probe microanalyzer with wavelength dispersive spectrometer: Two case reports. Respir Med Case Rep. 2016;18:66-72.

14. Chen HW, Su SF, Chien CT, Lin WH, Yu SL, Chou CC, et al. Titanium dioxide nanoparticles induce emphysema-like lung injury in mice. FASEB J. 2006;20: 2393-95.

15. Donaldson K, Stone V. Current hypotheses on the mechanisms of toxicity of ultrafine particles. Ann Ist Super Sanita. 2003: 39: 405-10.

16. Warheit DB, Hansen JF, Yuen IS, Kelly DP, Snajdr SI, Hartsky MA. Inhalation of high concentrations of low toxicity dusts in rats results in impaired pulmonary clearance mechanisms and persistent inflammation. Toxicol Appl Pharmacol. 1997; 145:10-22.

17. Garabrant D, Fine L, Oliver C, Bernstein L, Peters J. Abnormalities of pulmonary function and pleural disease among titanium metal production workers. Scand J Work Environ Health. 1987; 13: 47-51.

18. Moran C, Mullick F, Ishak K, Johnson, F, Hummer W. Identification of titanium in human tissues: probable role in pathologic processes. Hum Pathol. 1991; 2: 450-54.

19. Redline S, Barna B,Tomashefski J, Abraham, J. Granulomatous disease associated with pulmonary deposition of titanium. Br J Ind Med. 1986 Oct; $43(10): 652-6$.
20. Ellis ED, Watkins JP, Tankersley WG, Phillips JA, Girardi DJ. Occupational exposure and mortality among workers at three titanium dioxide plants. Am J Ind Med. 2013; 56(3):282-91.

21. Baan RA. Carcinogenic hazards from inhaled carbon black, titanium dioxide, and talc not containing asbestos or asbestiform fibers: recent evaluations by an IARC Monographs Working Group. Inhal Toxicol. 2007; 19: (Suppl 1) 213-228.

22. World Health Organization, International Agency for Research on Cancer. IARC monographs on the evaluation of carcinogenic risks to humans, vol. 93: Carbon black, titanium dioxide, nonasbestiform talc. Summaries and evaluations. Lyon: WHO; 2010. Disponible en: http://monographs. iarc.fr/ENG/ENG/Meetings/93titaniumdioxide.pdf.

23. Bañares MA, Haase A, Tran L, Lobaskin V, Oberdörster G, Rallo R, Leszczynski J, Hoet P, Korenstein R, Hardy B, Puzyn T. CompNanoTox2015: novel perspectives from a European conference on computational nanotoxicology on predictive nanotoxicology. Nanotoxicology. 2017; 11(7):839-845.

24. Vega E, Reyes E, Ruíz H, Garcia J, Sánchez, G, Martínez- Villa G, González U, Chow J, Watson G. 2004. Analysis of $\mathrm{PM}_{2.5}$ and $\mathrm{PM}_{10}$ in the atmosphere of Mexico City during 2000 -2002. J Air \& Waste Manage Assoc. 2004; 54: 786-98.

25. Alfaro-Moreno E, Martínez L, GarcíaCuellar C, Bonner JC, Murray CJ, Rosas I, et al. Biological effects induced in vitro by $\mathrm{PM}_{10}$ from three different zones of Mexico City. Environ Health Perspect. 2002; 110:715-720.

26. García A, Icaza G, Angeles F, Blanco $S$, GómezJ, DelgadilloM,etal.Proyectode monitoreo ambiental, microambiental y de exposición personal a partículas menores a diez micrómetros $\left(\mathrm{PM}_{10}\right)$ y menores a 2.5 micrómetros $\left(\mathrm{PM}_{25}\right)$ en la Zona Metropolitana de la Ciudad de México. Consejo de Estudios para la Restauración y Valoración Ambiental (CONSERVA), GDF 2000.

27. Rosas-Pérez I, Serrano J, Alfaro-Moreno E, Baumgardner D, Garcia-Cuellar C, Miranda C, et al. Relations between $\mathrm{PM}_{10}$ composition and cell toxicity: A multivariate and graphical Approach. Chemosphere. 2007; 67:1218-1228. 
28. Liebscher K, Smith H. Essential and nonessential trace elements. Arch Environ Health. 1968; 17:881-90.

29. Thompson K, Orvig C. Boon and bane of metal ions in medicine. Science. 2003; 300: 936-39.

30. Churg A, Stevens B, Wright JL. Comparison of the uptake of fine and ultrafine $\mathrm{TiO} 2$ in a tracheal explant system. Am J Physiol. 2003;274: 81- 86.

31. Bermúdez E, Mangum J, Asgharian B, Wong B, Reverdy E, Janszen D, et al. Long-term pulmonary responses of three laboratory rodent species to subchronic inhalation of pigmentary titanium dioxide particles. Toxicol Sci. 2002; 70:86-97.
32. Bermúdez E, Mangum J, Wong B, Asgharian B, Hext P, Warheit D, et al. Pulmonary responses of mice, rats, and hamsters to subchronic inhalation of ultrafine titanium dioxide particles. Toxicol Sci. 2004; 77: 347-57.

33. Liu H, Ma L, Zhao J, Liu J, Yan J, Ruan J, et al. Biochemical toxicity of nanoanatase $\mathrm{TiO} 2$ particles in mice. Biol Trace Elem Res. 2009; 129:170180.

34. Lovell M, Ehmann W, Markesbery W, Melethil S, Swyt C, Zatta P. Standardization in biological analyses of aluminum: what are the needs? J Toxicol Environ Health. 1996; 48: 637-48.

35. Farcal L, Torres-Andón F, Di Cristo L, Rotoli BM, Bussolati O, Bergamaschi
E, et al. Comprehensive In Vitro Toxicity Testing of a Panel of Representative Oxide Nanomaterials: First Steps towards an Intelligent Testing Strategy. PLoS ONE. 2015; 10(5): e0127174.

Correspondencia: Adelaida Manquián-Tejos Dirección: Centro Interdisciplinario de Investigaciones sobre el Medio Ambiente y Desarrollo (CIIEMAD), Instituto Politécnico Nacional de México. Calle 30 de Junio de 1520 s/n, Barrio La Laguna Ticoman, 07340 D.F., Mexico. PBX: 57296000 Extensión: 52703.

Correo electrónico:amanquiante@yahoo.es

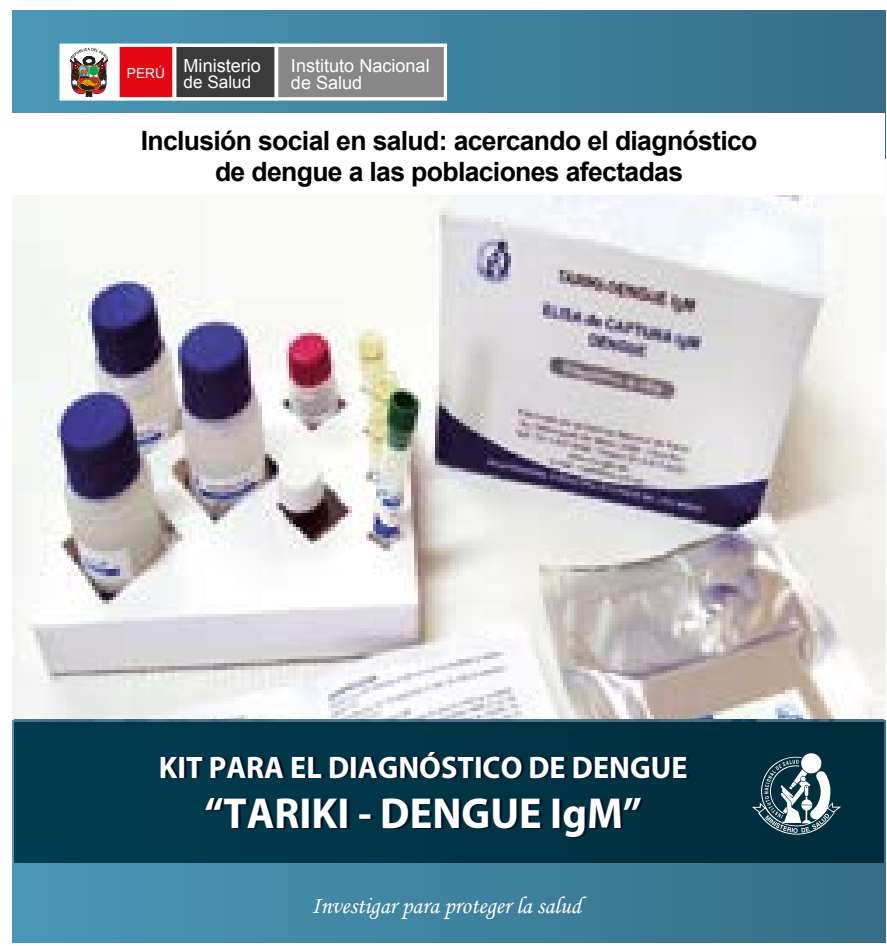

